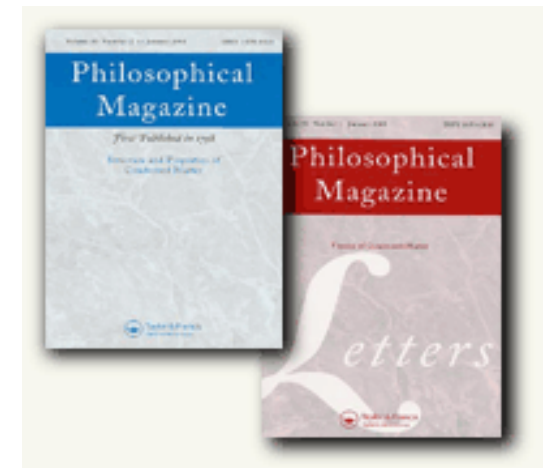

\title{
On the origin of large interstitial clusters in displacement cascades
}

\begin{tabular}{|c|c|}
\hline Journal: & Philosophical Magazine \& Philosophical Magazine Letters \\
\hline Manuscript ID: & TPHM-09-Apr-0175.R2 \\
\hline Journal Selection: & Philosophical Magazine \\
\hline $\begin{array}{r}\text { Date Submitted by the } \\
\text { Author: }\end{array}$ & 09-Jun-2009 \\
\hline Complete List of Authors: & $\begin{array}{l}\text { Calder, Andrew; University of Liverpool, Engineering } \\
\text { Bacon, David; University of Liverpool, Engineering } \\
\text { Barashev, Alexander; University of Liverpool, Engineering } \\
\text { Osetsky, Yuri; Oak Ridge National Laboratory, Materials Science } \\
\text { and Technology Division }\end{array}$ \\
\hline Keywords: & molecular dynamics, radiation damage \\
\hline Keywords (user supplied): & displacement cascades, self-interstitial atom clusters, shock waves \\
\hline
\end{tabular}

\section{S scholaroNE" Manuscript Central}


On the origin of large interstitial clusters in displacement cascades

\author{
A.F. Calder*†, D.J. Bacon $\dagger$, A.V. Barashev $\dagger$ and Yu.N. Osetskył \\ $\dagger$ Department of Engineering, The University of Liverpool, \\ Brownlow Hill, Liverpool, L69 3GH, UK \\ \$Computer Science and Mathematics Division, ORNL, \\ Oak Ridge, TN 37831-6138, USA
}

\begin{abstract}
Displacement cascades with wide ranges of primary knock-on atom (PKA) energy and mass in iron have been simulated by MD. New visualisation techniques are introduced to show how the shock-front dynamics and internal structure of a cascade develop over time. These reveal that the nature of the final damage is determined early on in the cascade process. $\mathrm{We}$ define a zone (termed 'spaghetti') in which atoms are moved to new lattice sites and show how it is created by a supersonic shock front expanding from the primary recoil event. A large cluster of self-interstitial atoms can form on the periphery of the spaghetti if a hypersonic recoil creates damage with a supersonic shock ahead of the main supersonic front. When the two fronts meet, the main one injects atoms into the low-density core of the other: these become interstitial atoms during the rapid recovery of the surrounding crystal. The hypersonic recoil occurs in less than $0.1 \mathrm{ps}$ after the primary recoil and the interstitial cluster is formed before the onset of the thermal spike phase of the cascade process. The corresponding number of vacancies is then formed in the spaghetti core as the crystal cools, i.e. at times one to two orders of magnitude longer. By using the spaghetti zone to define cascade volume, the energy density of a cascade is shown to be almost independent of the PKA mass. This throws into doubt the conventional energy-density interpretation of an increased defect yield with increasing PKA mass in ion irradiation.
\end{abstract}

Key words: radiation damage; displacement cascades; self-interstitial atom clusters; shock waves; molecular dynamics

*Author for correspondence. Email: afcalder@liv.ac.uk 


\section{Introduction}

Exposure to fast neutron irradiation in the core of a nuclear reactor can change the dimensions and mechanical properties of materials. These effects are due to microstructural changes that evolve with time and arise from the production of radiation damage. The primary damage is produced in displacement cascades by the recoil of primary knock-on atoms (PKAs) and consists of point defects and their clusters. The average number of vacancies and selfinterstitial atoms (SIAs) per cascade and the fraction of them created in clusters vary with PKA energy and mass, temperature and material. In recent years, much research in the field of radiation damage has focused on SIA clusters, in recognition of the fact that their thermal stability, one-dimensional mobility and interaction with impurity atoms are key elements in explaining several long-standing problems, and may provide a strong driving force for the damage accumulation [1].

Although the presence of small clusters of SIAs in displacement cascades has long been inferred from experiments, e.g. [2,3], it has not been possible to determine the mechanisms involved by direct experimental observation because the relevant length and time scales are measured in nanometres and picoseconds. Our present understanding of the formation and properties of defect clusters in cascades has largely been provided by molecular dynamics (MD) computer simulation. MD simulation of model crystals of sufficient size to contain cascades became possible in the late 1980s [4-8] due to increasing computer power and development of many-body interatomic potentials $[9,10]$, which provide realistic description of many important material properties yet offer computational efficiency. MD simulation has now become an invaluable source of information on cascades.

MD studies have shown that the number, $N_{\mathrm{F}}$, of vacancies or SIAs that survive intra-cascade recombination is fairly insensitive to metal and temperature. It increases with PKA recoil energy, $E_{\mathrm{PKA}}$, but, when expressed as a fraction of the NRT standard value [11], is approximately independent of $E_{\mathrm{PKA}}$ for energy above about $5 \mathrm{keV}[12,13]$. It is also insensitive to the presence of solute atoms, such as substitutional $\mathrm{Cu}[14,15]$ and $\mathrm{Cr}[16]$ and interstitial C [17] in $\alpha$-iron. This is because the number of defects produced in a cascade is governed by early stages of cascade development, when the atomic energies involved are much higher than the binding energy between atoms. The picture that has emerged regarding 
New insight into the cascade process has arisen as a by-product of another MD study of $\alpha$-Fe $[21,22]$, which in turn relates to experimental research undertaken on $\mathrm{Fe}$ and other metals some years ago, e.g. [23,24], and more recently [25,26]. In these experimental studies, selfion and heavy-ion irradiations of thin foils were used to investigate the effects of cascade parameters $E_{\mathrm{PKA}}$ and PKA mass, $m_{\mathrm{PKA}}$, on damage. Transmission electron microscope (TEM) examination of the foils showed that an increase of the incident ion mass increased the number of visible clusters formed per ion and the mean loop size. (The clusters were vacancy dislocation loops: SIA defects were assumed to be lost to the surfaces.) Thus, although $N_{\mathrm{F}}$ given by the NRT standard depends principally on $E_{\mathrm{PKA}}$ and the displacement threshold energy of the metal, PKA mass clearly affects defect production and clustering in cascades. In order to explain this, it was argued on the basis of binary-collision estimates obtained from [27], that the cascade volume is decreased by an increase in $m_{\mathrm{PKA}}$, with the result that higher energy density and vacancy concentration are achieved for the same $E_{\mathrm{PKA}}$. This produces a higher probability of cascade collapse and vacancy loop formation.

The PKA mass effect in bulk Fe has been investigated recently by MD simulation of cascades created by recoil of atoms with mass varying from $12 \mathrm{amu}$ (carbon) to $209 \mathrm{amu}$ (bismuth) and energy in the range $5-20 \mathrm{keV}$ [21]. It was found that large vacancy and SIA loops are produced with increasing frequency as PKA mass increases. These studies have now been extended to other conditions, and the structure and statistics of the cascade damage are the subject of another paper [22]. However, by using new visualisation techniques to probe the stages at which damage is formed in these high $m_{\text {PKA }}$ cascades, it became possible to reveal the mechanism by which large clusters of SIAs actually form early on in the cascade process. Furthermore, a meaningful definition of 'cascade volume' has emerged. The insight gained into the cascade process is another topic of this paper. 
Details of the MD simulations are first summarised in section 2 and then the results of a novel technique developed to visualise the displacement of atoms in a cascade are described with examples in section 3. As explained in section 4, the analysis assists understanding of the link between the dynamic processes associated with shock fronts created in the collision phase of cascades and the hot zone that characterises the thermal spike phase. This leads in section 5 to appreciation of the origin of large SIA clusters formed in cascades created by PKAs with high energy and mass. It is demonstrated in section 6 that the conventional energy-density arguments used to explain the formation of vacancy loops by cascade collapse are invalid. The conclusions are drawn in section 7.

\section{MD simulations}

The MD model of Fe was the same as that described in [21], but with a wider range of cascade parameters. In summary, it contained between 0.25 and 2 million atoms, depending on $E_{\mathrm{PKA}}$, which ranged from 5 to $30 \mathrm{keV}$. Atomic interactions were computed using the embedded-atom-method empirical potential from [28], which was developed with specific emphasis on correct description of the relative stability of SIAs of different configurations and the vacancy migration energy. The short-range $(\leq 0.1 \mathrm{~nm})$ pair part of the potential was replaced by the Universal screened-Coulomb potential [29] in order to better describe the high-energy atomic collisions that occur in cascades. The two potentials were joined smoothly by an exponential spline over the range $0.100-0.205 \mathrm{~nm}$. Electron-atom interactions were not included.

The crystal was equilibrated at $600 \mathrm{~K}$ prior to initiation of a cascade and maintained at constant volume with the lattice parameter, $a_{0}$, corresponding to the zero-pressure condition. Integration of the equations of motion of atoms was performed using a leapfrog algorithm with variable time-step, determined by limiting the displacement of the fastest atom to $0.003 a_{0}$ in one time-step. No temperature control was applied and the temperature increase due to introduction of the PKA was less than $70 \mathrm{~K}$.

Cascades in bulk Fe were simulated by giving one or more atoms velocity $\left(2 E_{\mathrm{PKA}} / m_{\mathrm{PKA}}\right)^{1 / 2}$ in either $<123>$ or $<135>$ crystallographic directions. Five different PKAs were used, namely C, 


\section{Spaghetti analysis and visualisation}

Unambiguous identification and cataloguing of point defects and their clusters is challenging when a large number of cascades has to be treated. Effort has tended to concentrate on two methods to identify damage. One is based on analysis of Wigner-Seitz (W-S) cells and is used to identify vacant sites (三 cells with no atoms) and SIAs (三 cells with more than one atom). This method is adequate for determining the number, $N_{\mathrm{F}}$, of surviving vacancies (equal to the number of surviving SIAs), but is not appropriate for identification of structures with correlated atomic displacements typical of stacking faults and interstitial dislocation loops with perfect Burgers vector [30]. The second method is displaced-atom (D-A) analysis, based on the position of atoms with respect to lattice sites. For each lattice site the closest distance to an atom is evaluated and if larger than a threshold value $\left(0.3 a_{0}\right.$ here $)$ the site is treated as vacant. Similarly, for each atom the closest distance to a lattice site is determined and, if larger than the threshold value, the atom is treated as 'displaced'. Evaluation of the number of point defects is not straightforward in this approach, either in the ballistic phase of a cascade, when many atoms undergo temporary elastic displacements, or at the end, when a dumbbell SIA is flagged as a vacant site and two displaced atoms, and a crowdion is revealed as a line of displaced atoms interspersed with one fewer vacant sites. Both methods were applied in the present work.

Figure 1 shows the variation of the number of displaced atoms calculated by the W-S cell and D-A methods versus time for a $20 \mathrm{keV} \mathrm{Bi}{ }_{3}$ cascade, for which $N_{\mathrm{F}}=77$. The number given by the D-A analysis is an order of magnitude larger at all times, for the reasons given above. The 
plots contain secondary peaks due to shock waves re-entering the MD cell through the periodic boundaries, although the W-S plot shows that these have no effect on the final damage. The regions (with labels) identified in Figure 1 are discussed in section 4.

[Insert Figure 1 about here]

To complement these two treatments of the vacancy and interstitial components of the primary cascade damage, we have also analysed the final coordinates of atoms in order to identify those that occupy lattice sites other than their original ones. To visualise the movement of atoms in this category, it has been found convenient to construct a line between the original and final sites of each atom. In a cascade involving permanent displacement of hundreds or thousands of atoms, nearly all site-to-site lines form a chain of connectors between sites in a sequence of atomic replacements, and there may be many such chains in the cascade zone. It should be noted that a chain is not the trajectory of a single atom. It is simply the path of many atomic replacements leading from a vacancy to a final SIA site. For want of a better word, we call such a set of chains 'spaghetti'. Some possibilities are illustrated schematically as follows.

Figure 2(a) shows a single chain starting at vacant site 'A' and ending at atom ' $E$ ', which is part of the single dumbbell SIA consisting of atoms ' $E$ ' and ' $G$ ' and vacant lattice site ' $F$ '. The atom originally at site 'A' was displaced to the site 'B', the atom originally at 'B' was displaced in turn to site 'C', and so on. Figure 2(b) shows two such chains, each of which gives rise to a Frenkel pair. Figure 2(c) presents a possibility in which three chains produce only two Frenkel pairs. Chains 2 and 3 both start with a vacancy and end at the same SIA, and chain 3 ends at the second SIA. Closed atomic replacement sequences, in which every atom replaces another and no defects are created, also occur.

[Insert Figure 2 about here]

The actual spaghetti zone and final damage arrangement produced by a $20 \mathrm{keV} \mathrm{Bi}_{2}$ cascade are presented in Figures 3(a) and (b), respectively. (The vacant sites and displaced atoms in these Figures are visualised by the D-A treatment. Most of the defects are obscured by the spaghetti in (a).) The site and direction of the PKA are indicated by the head and shaft of the arrow shown at the periphery of the zone at about the 10 o'clock position. This spaghetti is 
[Insert Figure 3 about here]

[Insert Figure 4 about here]

Samples of spaghetti and interstitial and vacancy damage (D-A visualisation) for $20 \mathrm{keV}$ cascades produced by $\mathrm{C}, \mathrm{Fe}$ and $\mathrm{Bi}_{2}$ PKAs are presented in Figures 5, 6 and 7, respectively. The principal features that stand out in these and similar Figures and their analysis are as follows.

[Insert Figures 5, 6 and 7 about here]

(i) The spaghetti zones in the light-mass C-PKA cascades (Figure 5) are diffuse and usually dispersed into sub-zones. These events with small $m_{\mathrm{PKA}}$ produce a significant proportion of their interstitials as single SIAs outside the spaghetti regions. An atom in one of these defects is joined to its parent spaghetti by short links associated with migration of the SIA, but the continuation of the chain to a vacant site within the spaghetti may be complex.

(ii) The spaghetti produced by the high-mass $\mathrm{Bi}_{2}$ PKAs (Figure 7) is denser and rarely exhibits sub-zones. The number of single SIAs linked by collision and migration chains to the main zone is relatively small for these cascades. The PKA event is always at the edge of the zone. Vacancies are created within the spaghetti and SIAs are formed at its periphery.

(iii) The self-ion cascades (Figure 6) tend to fall between the extremes of (i) and (ii). Fewer spaghetti sub-zones are formed than in the C-PKA cascades, but the probability of a single-zone cascade producing a sizeable SIA cluster is less than in the high- $m_{\mathrm{PKA}}$ damage. 
(iv) The pure atomic mixing chains, i.e. those with no SIAs and vacancies, constitute only a small fraction of all displaced atoms. Estimates from several cascades suggest less than $10 \%$.

(v) It is clear from the $N_{\mathrm{F}}$ data included in Figures 4 to 7 that a large amount of spaghetti is not necessarily associated with a large number of Frenkel pairs.

(vi) The probability of formation of clusters of interstitial atoms increases with increasing PKA mass. (The nature of these clusters and the vacancy arrangements within the spaghetti zone core is the topic of another paper [22].) However, the creation of a dense spaghetti does not guarantee formation of a cluster. Thus, the formation of defects requires conditions other than simple deposition of energy, which is revealed in the huge mixing shown by the spaghetti. Clustering of SIAs increases defect survival and the conditions leading to clustering are discussed in the next section.

\section{Cascade dynamics}

With reference to the plot in Figure 1, the rapidly expanding zone of displaced atoms exhibits two distinct stages before the number of displaced atoms reaches the peak at time $t \sim 1 \mathrm{ps.} \mathrm{Up}$ to $t \sim 0.1 \mathrm{ps}$, the number of displaced atoms in the D-A treatment is approximately proportional to $t^{3}$ (dotted line), implying a zone front moving with approximately constant velocity. Estimates obtained from either visualisations of displaced atoms or the change in volume of the zone with time show that the front moves outwards with a speed of approximately $30 \mathrm{~km} \mathrm{~s}^{-1}$, i.e. about five times the longitudinal velocity of sound, $v_{\mathrm{s}}$, in iron. With the elastic constants calculated for the potential used here, $v_{\mathrm{s}}$ varies 5.6 to $6.5 \mathrm{~km} \mathrm{~s}^{-1}$ at $0 \mathrm{~K}$ for different low index directions. The variation is 5.1 to $6.1 \mathrm{~km} \mathrm{~s}^{-1}$ with the experimental elastic constants measured at 595K [31]. Thus, the front is supersonic in character, i.e. speed in the range $v_{\mathrm{s}}$ to $5 v_{\mathrm{s}}$, up to $t \sim 0.1 \mathrm{ps}$, but becomes sonic as $t$ increases further to $1 \mathrm{ps.} \mathrm{More}$ information on these supersonic and sonic shock fronts follows later in this sub-section.

In the context of the present paper, W-S visualisation of interstitial atoms and vacancies of some cascades reveals features that are characteristic of interstitial cluster formation. Figure 8 contains images generated by the $\mathrm{W}-\mathrm{S}$ visualisation method of a $30 \mathrm{keV} \mathrm{Bi}_{2}$ cascade at times from 0.04 to $32.6 \mathrm{ps}$, as indicated. The image in Figure 8(a) for $t=0.0402$ ps shows that two hypersonic recoil (HR) events labelled HR1 and HR2 emerge ahead of the main zone of 
[Insert Figure 8 about here]

The terminology shock front implies an abrupt change in pressure, density and temperature. We have examined the latter two parameters in the following way. Figure 9 contains snapshot cross-sections through the central region of the cascade in Figure 8 at various times from 0.04 to $3.50 \mathrm{ps}$. The cross-section plane is $(01 \overline{1})$ and the direction of the abscissa and ordinate is [100] and [011], respectively. The scale unit of length is $a_{0}$. The colour (grey shading in the printed version of the paper) indicates the temperature associated with each atom lying within $0.7 a_{0}$ of the plane of the Figure, as shown in the scale in Figure 9(a). For an atom with kinetic energy $E_{\mathrm{k}}$, the temperature, $T$, is given by $T=2 E_{\mathrm{k}} / 3 k_{\mathrm{B}}$, where $k_{\mathrm{B}}$ is the Boltzmann constant. To smooth extreme variations in the temperature from atom to atom, the temperature in the Figures is the average over all atoms in a sphere centred on the atom in question. A sphere radius of $1.1 a_{0}$ was found to provide reasonable sensitivity in the temperature plots. In a perfect crystal, a sphere of this radius contains the central atom and its 14 first- and second-nearest neighbours.

[Insert Figure 9 about here] 
The sequence of plots in Figure 9 show that, for $t$ up to about $0.1 \mathrm{ps,} T$ has an almost discontinuous change at the supersonic front between the cascade core and the surrounding crystal. Behind the front, crystalline order is destroyed and atoms have temperature in excess of $2000 \mathrm{~K}$, which is just above the melting point of $1750 \pm 25 \mathrm{~K}$ for the interatomic potential used [32]. This hot core zone also has regions near the centre with no atoms. The shock front becomes less abrupt and its velocity slows by $t=0.2$ ps (Figure 9(c)), beyond which there is noticeable diffusion of thermal energy into the surrounding crystal. The 'molten' zone reaches its maximum size by 0.3 to $0.4 \mathrm{ps}$, which is much shorter than the time of the peak in the number of displaced atoms in Figure 1, but it only starts to shrink after 3 to 4 ps. The region of spaghetti defined in section 3 corresponds to this zone at its maximum size. This description of a highly disordered, hot cascade core is consistent with the cross-sections showing atomic positions produced from early computer simulations of displacement cascades, e.g. [4-6].

Another way to visualise the shock-wave nature of the disturbance created by a cascade is to consider the atomic density. This is achieved in Figure 10 by plots showing the atomic neighbour density in the same cross-section and at the same times as those in Figure 9. The neighbour density of each atoms is defined to be the number of atoms in a sphere centred on the atom in question. A sphere radius of $1.405 a_{0}$ was found to be suitable because it excludes the twelve third-nearest neighbour atoms at a distance of $1.414 a_{0}$ in a prefect crystal, but is sufficiently close to it to enable increases in density associated with shock effects to be detected. The neighbour density is seen to be high (exceeding 26 at some sites) at the shock front, which is compensated by very low values (between 0 and 8) in the central core of the cascade. The front defined by density is more obvious than that in Figure 9 for time greater than about $0.2 \mathrm{ps}$, when it has sonic speed and the deformation associated with it is purely elastic.

\section{[Insert Figure 10 about here]}

It was noted above that W-S identification of displaced atoms reveals two hypersonic collision events created ahead of the supersonic front in this cascade. They are apparent as two 'ears' at about one o'clock in Figures 9(a) and (b), and appear to be subsumed within the zone behind the main front in Figure 9(c). However, Figure 10 shows that they actually retain a separate identity and create their own shock fronts before being reached by the main front 
(parts (c) and (d)). This is important for the formation of large clusters of SIAs, as shown in the next section.

\section{On the origin of large SIA clusters}

As explained in section 1, the computer simulations in [21] showed that the probability of producing large vacancy and SIA clusters in a displacement cascade increases with increasing PKA mass and energy. This study has now been extended and results on the structure and statistics of the clusters are the subject of another paper [22]. The relevance of the present work is that by using the visualisation techniques described to examine cascade development and the final damage, it has been possible to observe a correlation between the production of large clusters of SIAs and a process initiated at a very early stage of cascade development. Correspondingly, the creation of large SIA clusters by events early on in the cascade process leads to the formation of large vacancy clusters by the end of the thermal spike phase, i.e. after many picoseconds.

We have observed that the formation of an SIA cluster that becomes distinguishable as such by the end of the collision phase, i.e. at times of a few picoseconds, proceeds by creation of a hypersonic energetic recoil atom that travels beyond the main shock front and creates its own shock wave and associated damage near the edge of what later becomes the spaghetti zone. In other words, the site for the cluster is created in less than $0.1 \mathrm{ps}$ from the time of the initial primary recoil. (The time when the hypersonic recoil stops is indicated in Figure 1). The process is summarised schematically in Figures 11(a)-(d). Most of the supersonic shock front does not encounter damage formed by a hypersonic event and the atoms displaced by the front outside the spaghetti zone simply return to their lattice sites by elastic relaxation after the time of the peak in Figure 1. However, when an energetic impact event produces one or more hypersonic recoils (two are shown in Figure 11(a)) beyond the main front (see Figure 11(b)), the main and secondary supersonic shock waves with high atomic density at their fronts and low density disorder behind expand and meet (Figure 11(c)). It appears from the evidence of the visualisation techniques that the main front then injects atoms into the core of the secondary region(s) (Figure 11(d)). The number of atoms there exceeds the number of lattice sites and the excess atoms become trapped by the rapid recovery of the surrounding crystal (Figure 11(e)) and form an interstitial cluster. (Note that it seems unlikely that interstitial atoms form in the compression region where the two fronts meet, because the Burgers vectors 
of the interstitial dislocation loops that eventually form are found to be tangential to the spaghetti zone [22], as depicted in Figure 11(d).) The interstitial atoms formed in this way are matched by vacancies formed during solidification of the hot disordered core of the main zone, which is a relatively slow process. It was inferred from atomic configuration crosssections produced in early simulations of cascades in fcc metals that free volume accumulates on the liquid side of the solidification interface, with the result that a vacancy cluster forms in the centre of the cascade zone, e.g. [4-6].

\section{[Insert Figure 11 about here]}

It should be noted that the formation of dislocation loops seen here is not the same as the prismatic loop punching mechanism postulated by Diaz de la Rubia and Guinan [33], which would produce loops with Burgers vector orientated radially from the cascade core. Nor does it appear to be related to the mechanism of shear dislocation loop creation behind planar shock fronts produced by impact. Germann et al. [34] and Kadau et al. [35] have used MD to simulate the effects of a planar shock front in models of fcc and bcc crystals, and interpreted the dislocation structure observed in terms of earlier models of shock effects [36-38]. The dislocations were created in the strained crystalline material behind the front, which is not the process found in the present work. In the simulations of iron crystals, Kadau et al. [35] found that solid-solid, martensitic-like transformations were induced by shock. Transformations were not present in the cascade damage regions here, presumably because the melt-like zone has a long enough life and is sufficiently small for it to solidify coherently with the surrounding bec crystal.

Thus, we have established a connection between formation of large SIA clusters and the existence of a high-energy, hypersonic recoil events in the primary stage of cascades. We first became aware of the connection through visualising effects in cascades due to PKAs with high mass, and it then became apparent in all the cascade simulations that resulted in large clusters. It seems essential that the hypersonic recoil atom stops beyond, but near, the edge of the spaghetti region. If it stops well beyond the supersonic front, it results in sub-cascade that develops independently of the main cascade. If it were within the vacancy-rich cascade core, where the energy deposited is much higher and the lifetime of such an excitation is long, the damage would be effectively annealed. 


\section{On energy density arguments in cascade collapse}

It was noted in section 1 that the effects of parameters $E_{\mathrm{PKA}}$ and $m_{\mathrm{PKA}}$ on cascade damage on Fe and other metals, e.g. [23-26], have been investigated experimentally by ion irradiation of thin foils and subsequent examination of the visible defects, i.e. dislocation loops, in a TEM. This has shown that increasing ion mass increases the number of visible clusters per ion and the mean cluster size. Thus, although $N_{\mathrm{F}}$ given by the NRT standard depends principally on $E_{\mathrm{PKA}}, \mathrm{PKA}$ mass clearly affects defect production and clustering in cascades. In order to explain this, it is generally argued on the basis of binary-collision estimates that the cascade volume is decreased by an increase in $m_{\mathrm{PKA}}$, with the result that higher energy density and vacancy concentration are achieved for the same $E_{\mathrm{PKA}}$. This produces a higher probability of cascade collapse. Note that collapse creates vacancy loops late on in the cascade process, not interstitial loops. SIA defects are assumed to be lost to the nearby surfaces in these thin-foil experiments.

The present work offers a different explanation for the increasing probability of formation of visible vacancy loops with increasing PKA mass. The supersaturation of vacancies in the cascade core, which is necessary for cascade collapse to a vacancy loop, has been shown to arise from the prior clustering of interstitial atoms, which is initiated by a hypersonic recoil process in less than $0.1 \mathrm{ps}$ from the time of the primary recoil event. Furthermore, the techniques of visualisation presented here allow the concept of 'cascade volume' and 'energy density' to be considered from a more realistic standpoint than that adopted hitherto. The spaghetti analysis permits us to define an effective region within which permanent damage occurs, and then calculate its volume and, hence, energy density.

Calculation of the volume was performed using the MSMS code of Sanner, Spehner and Olson [39], which is normally used to calculate molecular surfaces and the solvent-accessible surface (SAS) and solvent-excluded surface (SES) of large protein and nucleic acid molecules. For the cascade calculations it was assumed that the spaghetti region consists of a set of spheres representing iron atoms centred on the 'joints' in the spaghetti chains with radius $r_{\mathrm{s}}$ and a MSMS probe sphere radius of radius $r_{\mathrm{p}}$, as shown schematically in Figure 12(a). The SES topological boundary was chosen as the appropriate surface of the spaghetti region surface area and associated enclosed volume. Values $r_{\mathrm{s}}=0.55 a_{0}$, i.e. slightly larger than the hard-sphere atomic radius of $0.42 a_{0}$, and $r_{\mathrm{p}}=0.6 a_{0}$ were found to be suitable, although some 
surfaces required a slight adjustment to $r_{\mathrm{p}}$ to allow the SES to be computed successfully. If spatially-separated SES volumes were identified for a single cascade event, the sum of all the identified SES areas and volumes was taken. Visualisations of the MSMS-computed SES were done using PyMOL [40] and a Python script msms_cgo.py from [41]. (PyMOL was also used for the D-A, W-S and spaghetti images shown in this paper.) An example for the cascade of Figures 8-10 is presented in Figure 12(b), which is a superposition of the interstitial atoms and vacancies using W-S visualization, the spaghetti and the MSMS probe spheres. It can be seen that the volume and surface area include the main body of spaghetti as well as chains that lead to single SIAs (e.g. top right of figure) and simple replacement sequences that emerge from, and return to, the main body. For cascades with high $m_{\mathrm{PKA}}$, the contribution of such chains is small.

[Insert Figure 12 about here]

Figure 13 contains mean values (with bars representing the standard deviation) for the area, $A_{\mathrm{s}}$, and volume, $V_{\mathrm{s}}$, of the spaghetti zone of all the cascades for each of the combinations of $E_{\mathrm{PKA}}$ and $m_{\mathrm{PKA}}$ considered here (619 cascades in total). Several features stand out. First, $V_{\mathrm{s}}$ depends approximately linearly on the PKA energy. Second, the surface area of the region of permanent damage is seen to be sensitive to the PKA mass and decreases with increasing mass. This reflects the more irregular shape when $m_{\mathrm{PKA}}$ is reduced. Third, and most important, the cascade volume, and hence energy density, is almost independent of PKA mass. This is in stark contrast to the conventional understanding derived from binary collision models, and leads to the conclusion that interpretation of trends from experimental measurements of vacancy defect yield based on energy density arguments is not applicable for the analysis of cascade collapse.

[Insert Figure 13 about here]

\section{Conclusions}

1. The new visualisation techniques introduced here show how the shock-front dynamics and internal structure of a displacement develop over time and that the nature of the final damage is determined early on in the ballistic phase. 
2. The spaghetti zone in which atoms are moved to new lattice sites by the cascade process is identified with a hot, disordered zone created as a supersonic shock front. The boundary of the spaghetti coincides with the transition region where the speed of the front slows to the sonic regime.

3. In some cascades, a large cluster of self-interstitial atoms is formed on the periphery of the spaghetti. This is the result of interaction between two kinetic elements, namely a hypersonic recoil that occurs in less than 0.1 ps after the primary recoil and creates damage with its attendant supersonic shock wave ahead of the main supersonic front. When the two shock waves meet, the main one injects atoms into the low-density core of the other. The number of atoms there then exceeds the number of lattice sites available during the rapid cooling and elastic recovery of the surrounding crystal.

4. Large interstitial clusters are therefore formed before the onset of the thermal spike phase, i.e. in less than a few tenths of a picosecond after the primary recoil. A correspondingly large number of vacancies forms later in the core of the spaghetti as the crystal lattice is restored during cooling, i.e. at times one to two orders of magnitude longer.

5. By defining cascade volume in terms of the permanently damaged spaghetti region, the energy density of a cascade is shown to be independent of the PKA mass. This result rules out the conventional energy-density interpretation of the increased probability of defect cluster formation with increasing PKA mass observed after ion irradiation.

\section{Acknowledgments}

The research was supported by grant GR/S81162/01 from the UK Engineering and Physical Sciences Research Council, and partly by the Division of Materials Sciences and Engineering and the Office of Fusion Energy Sciences, U.S. Department of Energy, under contract DEAC05-00OR22725 with UT-Battelle, LLC. 


\section{References}

[1] B.N. Singh, M. Eldrup, A. Horsewell, et al., Philos. Mag. A 80 (2000) p.2629.

[2] B. von Guerard, D. Grasse and J. Peisl, Phys. Rev. Lett. 44 (1980) p.262.

[3] R. Rauch, J. Peisl, A. Schmalzbauer, et al., J. Nucl. Mater. 168 (1989) p.101.

[4] T. Diaz de la Rubia and M.W.Guinan, J. Nucl. Mater. 174 (1989) p.151.

[5] T. Diaz de la Rubia, R.S. Averback, H. Hsieh, et al., J. Mater. Res. 4 (1989) p.579.

[6] R.S. Averback, H. Hsieh, T. Diaz de la Rubia, et al., J. Nucl. Mater. 179-181 (1991) p.87.

[7] A.J.E. Foreman, W.J. Phythian and C.A. English, Philos. Mag. A 66 (1992) p.671.

[8] A.F. Calder and D.J. Bacon, J. Nucl. Mater. 207 (1993) p.25.

[9] M.W. Finnis and J.E. Sinclair, Philos. Mag. A 50 (1984) p.45.

[10] M.S. Daw and M.I. Baskes, Phys. Rev. B 29 (1984) p.6443.

[11] M. J. Norgett, M. T. Robinson and I. M. Torrens. Nucl. Eng. and Design 33 (1975) p.50.

[12] D.J. Bacon, A.F. Calder, F. Gao, et al., Nucl. Instrum. and Meth. B 102 (1995) p.37.

[13] D.J. Bacon, F. Gao and Yu.N. Osetsky, J. Computer-Aided Des. 6 (1999) p.225.

[14] A.F. Calder and D.J. Bacon, Mater. Res. Soc. Symp. Proc. 439 (1997) p.521.

[15] C.S. Becquart, C. Domain, J.C. Van Duysen, et al., J. Nucl. Mater. 294 (2001) p.274.

[16] D.A. Terentyev, L. Malerba, R. Chakarova, et al., J. Nucl. Mater. 349 (2006) p.119.

[17] A.F. Calder, D.J. Bacon, A.V. Barashev, et al., J. Nucl. Mater. 382 (2008) p.91.

[18] D.J. Bacon, F. Gao and Yu.N. Osetsky, J. Nucl. Mater. 276 (2000) p.1.

[19] Yu.N. Osetsky, D.J. Bacon and B.N. Singh, J. Nucl. Mater. 307-311 (2002) p.866.

[20] D. J. Bacon, Yu. N. Osetsky, R. Stoller, et al., J. Nucl. Mater. 323 (2003) p.152.

[21] A.F. Calder, D.J. Bacon, A.V. Barashev, et al., Philos. Mag. Lett. 88 (2008) p.43.

[22] A.F. Calder, D.J. Bacon, A.V. Barashev, et al., in preparation.

[23] M.L. Jenkins, C.A. English and B.L. Eyre, Philos. Mag. A 38 (1975) p.97.

[24] C.A. English and M.L. Jenkins, Mat. Sci. Forum 15-18 (1987) p.1003.

[25] Z. Yao, M. Hernández-Mayoral, M.L. Jenkins, et al., Philos. Mag. 88 (2008) p.2851.

[26] M.L. Jenkins and C.A. English, this issue.

[27] K.B. Winterbon, P. Sigmund and J.B. Sanders, Matematisk-Fysiske Meddelelser Udgivet af det Kongelige Danske Videnskabernes Selskab 37 (1970) p.1.

[28] G.J. Ackland, M.I. Mendelev, D.J. Srolovitz, et al., J. Phys.: Condens. Matter 16 (2004) p.S2629. 
[29] J.P. Biersack and J.F. Ziegler, Nucl. Instrum. and Meth. 194 (1982) p.93.

[30] R.E. Voskoboinikov, Yu.N. Osetsky and D.J. Bacon, J. Nucl. Mater. 377 (2008) p.385.

[31] A. E. Lord Jr. and D. N. Beshers, J. Appl. Phys. 36 (1965) p.1620

[32] C. Björkas and K. Nordlund, Nucl. Instr. Meth. Phys. Res. B. 259 (2007) p.853.

[33] T. Diaz de la Rubia and M.W.Guinan, Phys. Rev. Lett. 66 (1991) p.2766.

[34] T.C. Germann, D. Tanguy, B.L. Holian, et al., Metall. and Mater. Trans. A 35A (2004) p.2609.

[35] K. Kadau, T.C. Germann, P.S. Lomdahl, et al., Phys. Rev. B 72 (2005) 064120.

[36] C.S. Smith, Trans. AIME 212 (1958) p.574.

[37] E. Hornbogen, Acta Metall. 10 (1962) p.978.

[38] R.W. Armstrong, R.S. Miller and H.W. Sandusky, ONR Workshop on Dynamic Deformation, Fracture, and Transient Combustion, Chemical Propulsion Information Agency Publication 474, Chemical Propulsion Information Agency, Chestertown, MD, (1987) p.77.

[39] M.F. Sanner, A.J. Olson and J-C. Spehner, Biopolymers 38 (1996) p.305.

[40] W.L. De Lano, The PyMOL Molecular Graphics System, DeLano Scientific LLC, Palo Alto, CA, USA (2008). <http://www.pymol.org>

[41] R.L. Campbell, Queen's University, Kingston, ON, Canada. $<$ http://pldserver1.biochem.queensu.ca/ rlc/work/pymol/> 


\section{Figure captions}

Figure 1. Variation of the number of displaced atoms calculated by the W-S cell and D-A methods versus time for a $20 \mathrm{keV} \mathrm{Bi}_{3}$ cascade.

Figure 2. Schematic representation of different types of chains of the spaghetti.

Figure 3. (a) The actual spaghetti zone, (b) final damage arrangement produced by a $20 \mathrm{keV}$ $\mathrm{Bi}_{2}$ cascade and (c) schematic representation of chains. The vacant sites and displaced atoms are visualised by the D-A treatment.

Figure 4. Visualisations of all eight chains of the spaghetti presented in Figure 3(c).

Figure 5. Sample of spaghetti and interstitial and vacancy damage (D-A visualisation) for $20 \mathrm{keV}$ cascades produced by C PKA.

Figure 6. Same as in Figure 5 but for Fe PKA.

Figure 7. Same as in Figure 5 but for $\mathrm{Bi}_{2}$ PKA.

Figure 8 . W-S visualisation snapshots at various times of a cascade created by a $30 \mathrm{keV} \mathrm{Bi}_{2}$ PKA. HR1 and HR2 in (a) are hypersonic recoils that emerge ahead of the primary shock front and result in the formation of the large SIA cluster indicated in (f). The spaghetti zone and the W-S image are superimposed in (e).

Figure 9. Snapshot cross-sections through the cascades in Figure 8 at various times. The cross-section plane is $(01 \overline{1})$ and the direction of the abscissa and ordinate is [100] and [011], respectively. The scale unit of length is $a_{0}$. The colour (grey shading in the printed version of the paper) indicates the temperature associated with each atom lying within $0.7 a_{0}$ of the plane of the paper, as shown in the scale in the part (a). The temperature is the average over atoms in a sphere of radius $r_{\mathrm{c}}$ $=1.1 a_{0}$ centred on the atom in question.

Figure 10. The atomic neighbour density (the number of atoms in a sphere of radius $r_{\mathrm{c}}=$ $1.405 a_{0}$ ) centred on the atom in question in the same cross-section and at the same times as those in Figure 9.

Figure 11. Schematic representation of cascade development over time and interstitial cluster formation.

Figure 12. (a) Schematic illustration of the MSMS construction used to calculate the surface area and volume associated with a spaghetti chain. (b) Superposition of the interstitial atoms and vacancies using W-S visualization, the spaghetti and the MSMS probe spheres for the cascade shown in Figures 8-10. 
1

2

3

4

5

6

7

8

9

10

11

12

13

14

15

16

17

18

19

20

21

22

23

24

25

26

27

28

29

30

31

32

33

34

35

36

37

38

39

40

41

42

43

44

45

46

47

48

49

50

51

52

53

54

55

56

57

58

59

60

Figure 13. Mean values with bars representing the standard error for the area, $A_{\mathrm{s}}$, and volume, $V_{\mathrm{s}}$, of the spaghetti zone of all the cascades for each of the combinations of $E_{\mathrm{PKA}}$ and $m_{\mathrm{PKA}}$ considered here. 


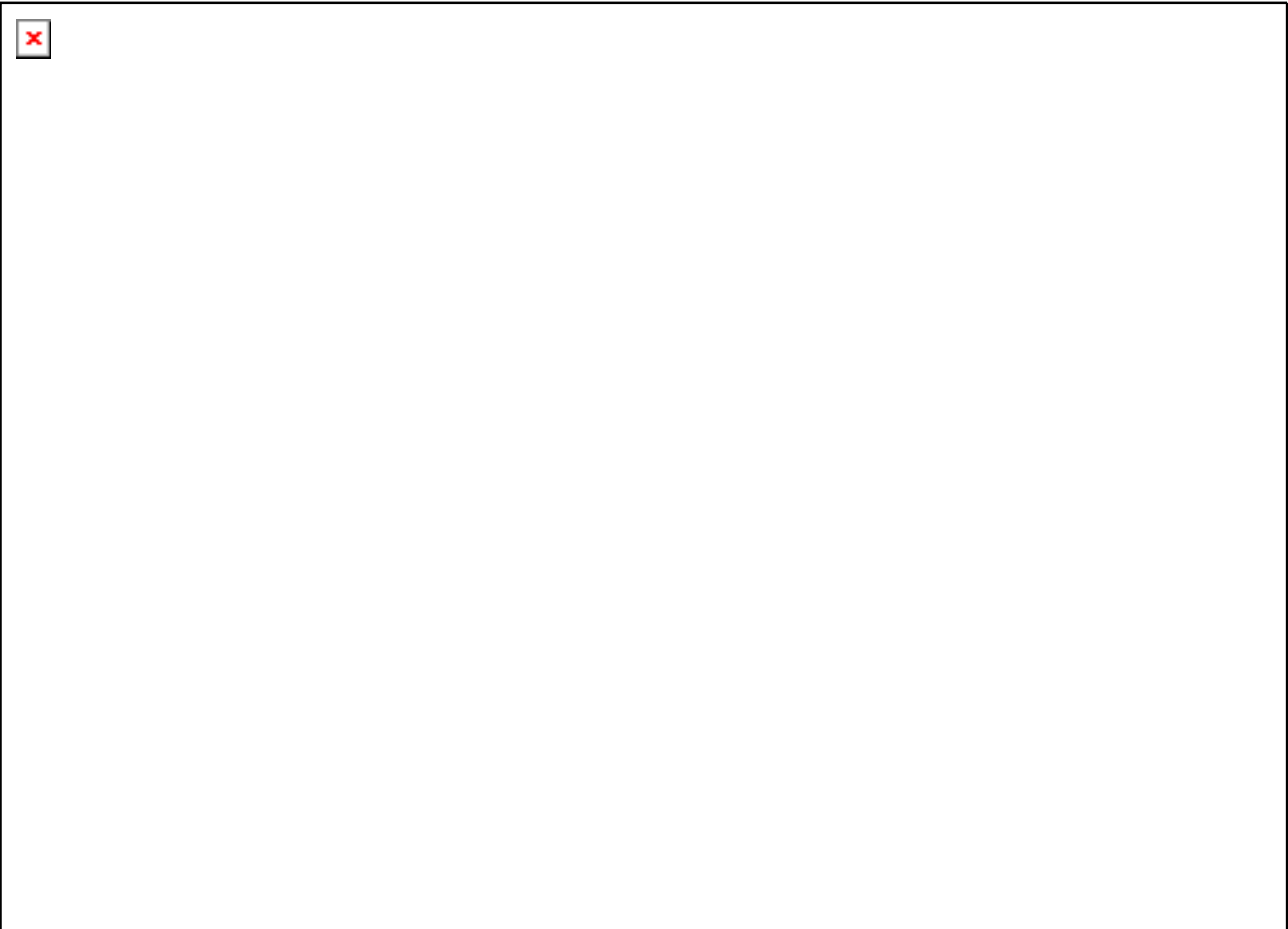

Figure 1 $101 \times 73 \mathrm{~mm}(300 \times 300$ DPI $)$ 


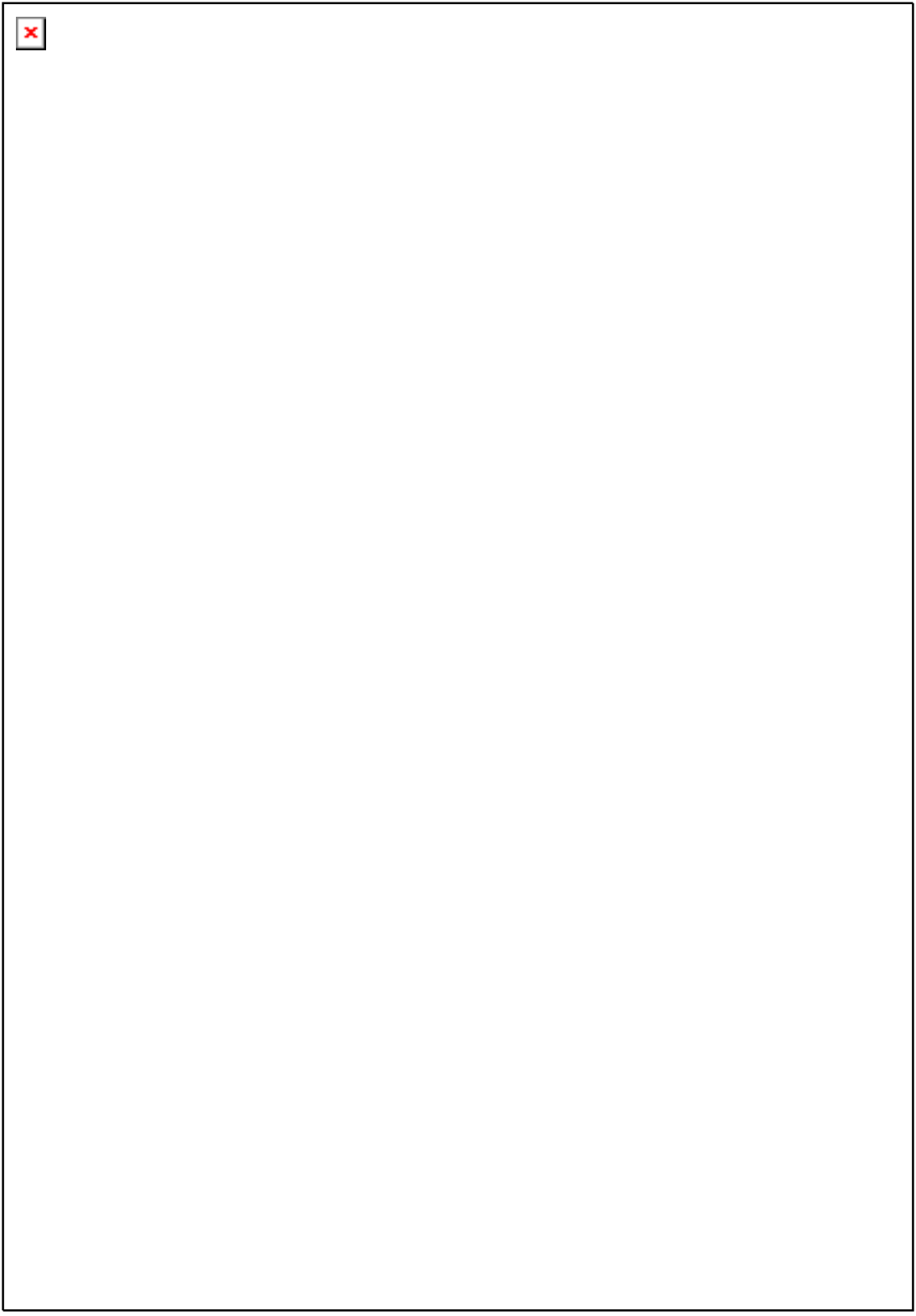

Figure 2 $118 \times 169 \mathrm{~mm}$ ( $300 \times 300$ DPI) 


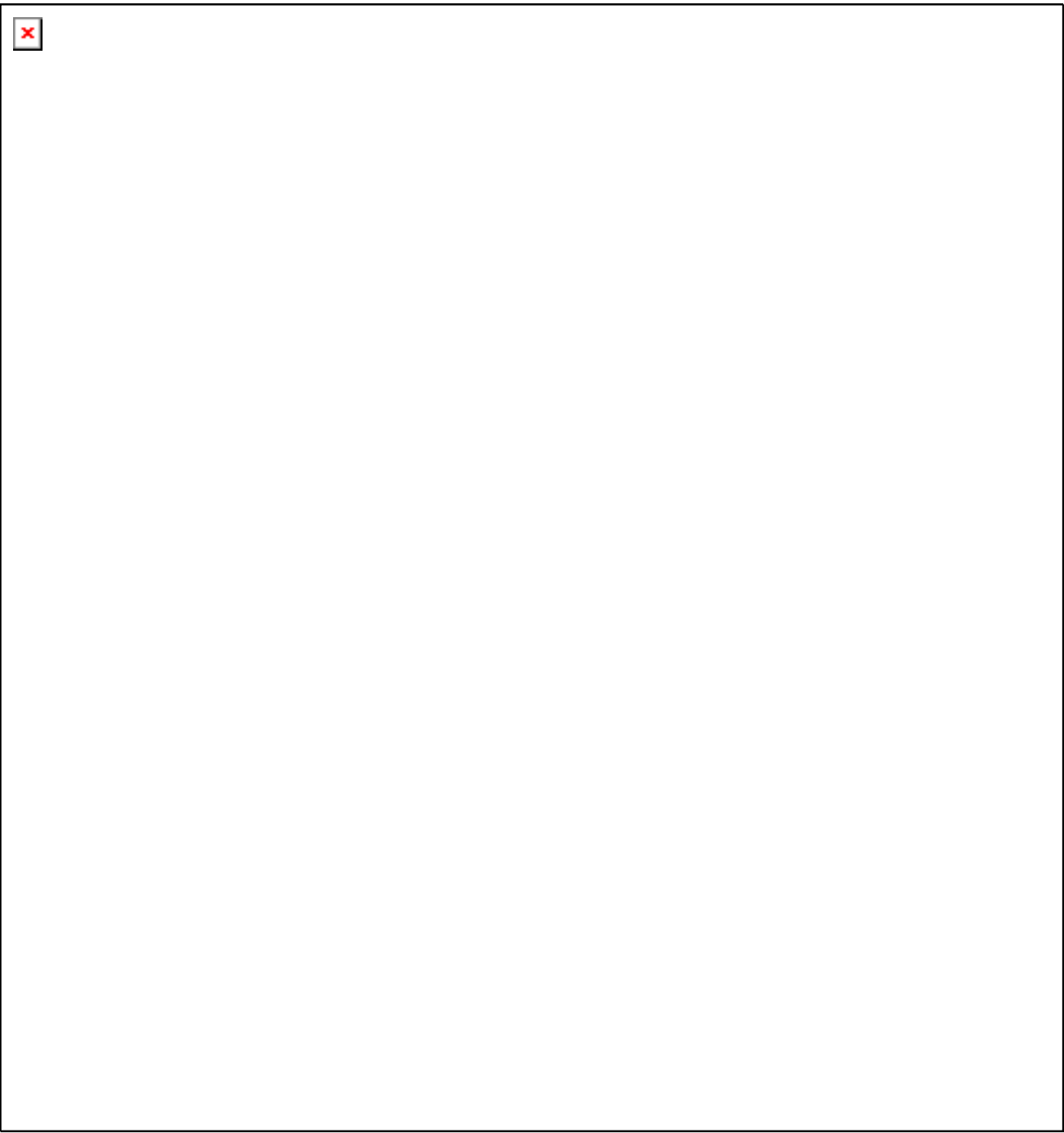

Figure 3

$199 \times 211 \mathrm{~mm}(400 \times 400 \mathrm{DPI})$ 


1
2
3
4
5
6
7
8
9
10
11
12
13
14
15
16
17
18
19
20
21
22
23
24
25
26
27
28
29
30
31
32
33
34
35
36
37
38
39
40
41
42
43
44
45
46
47
48
49
50
51
52
53
54
55
56
57
58
60

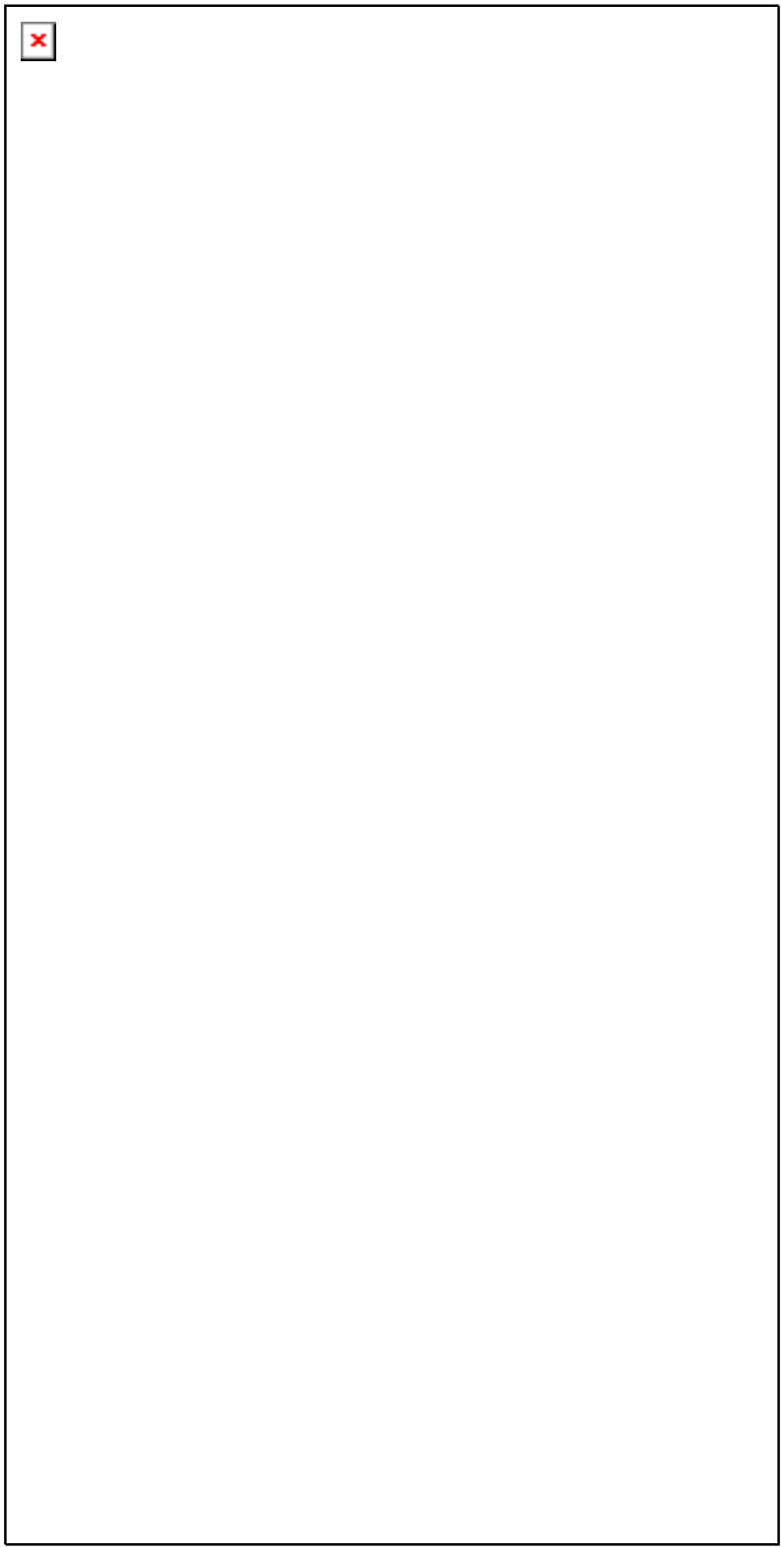

Figure 4 $139 \times 277 \mathrm{~mm}(400 \times 400 \mathrm{DPI})$ 


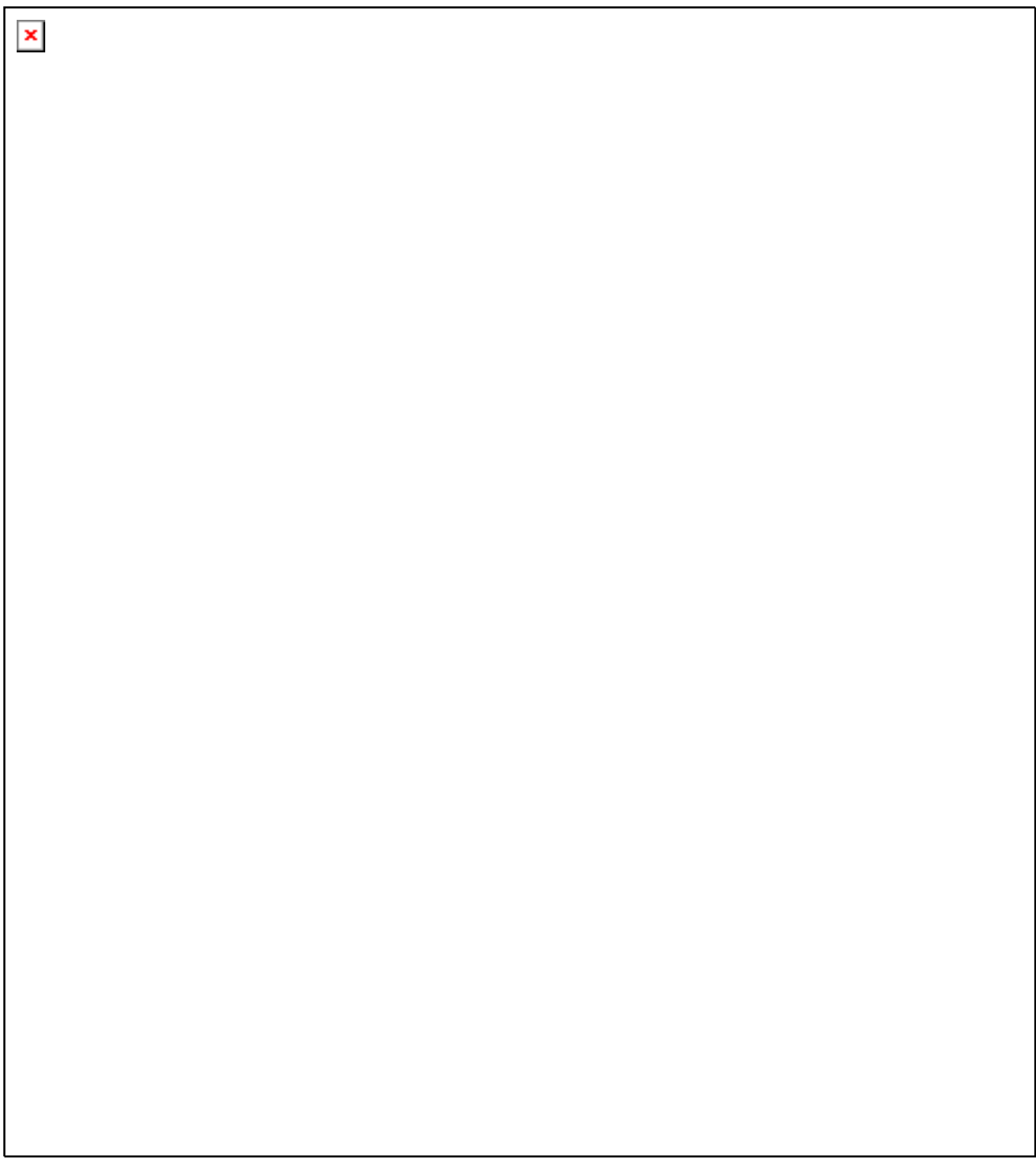

Figure 5 $198 \times 221 \mathrm{~mm}(600 \times 600 \mathrm{DPI})$

http://mc.manuscriptcentral.com/pm-pml 


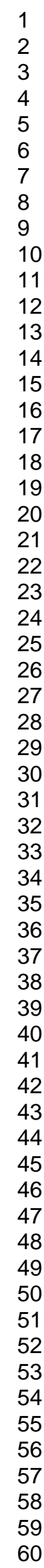

$\mathrm{x}$

Figure 6 $200 \times 221 \mathrm{~mm}(600 \times 600 \mathrm{DPI})$ 


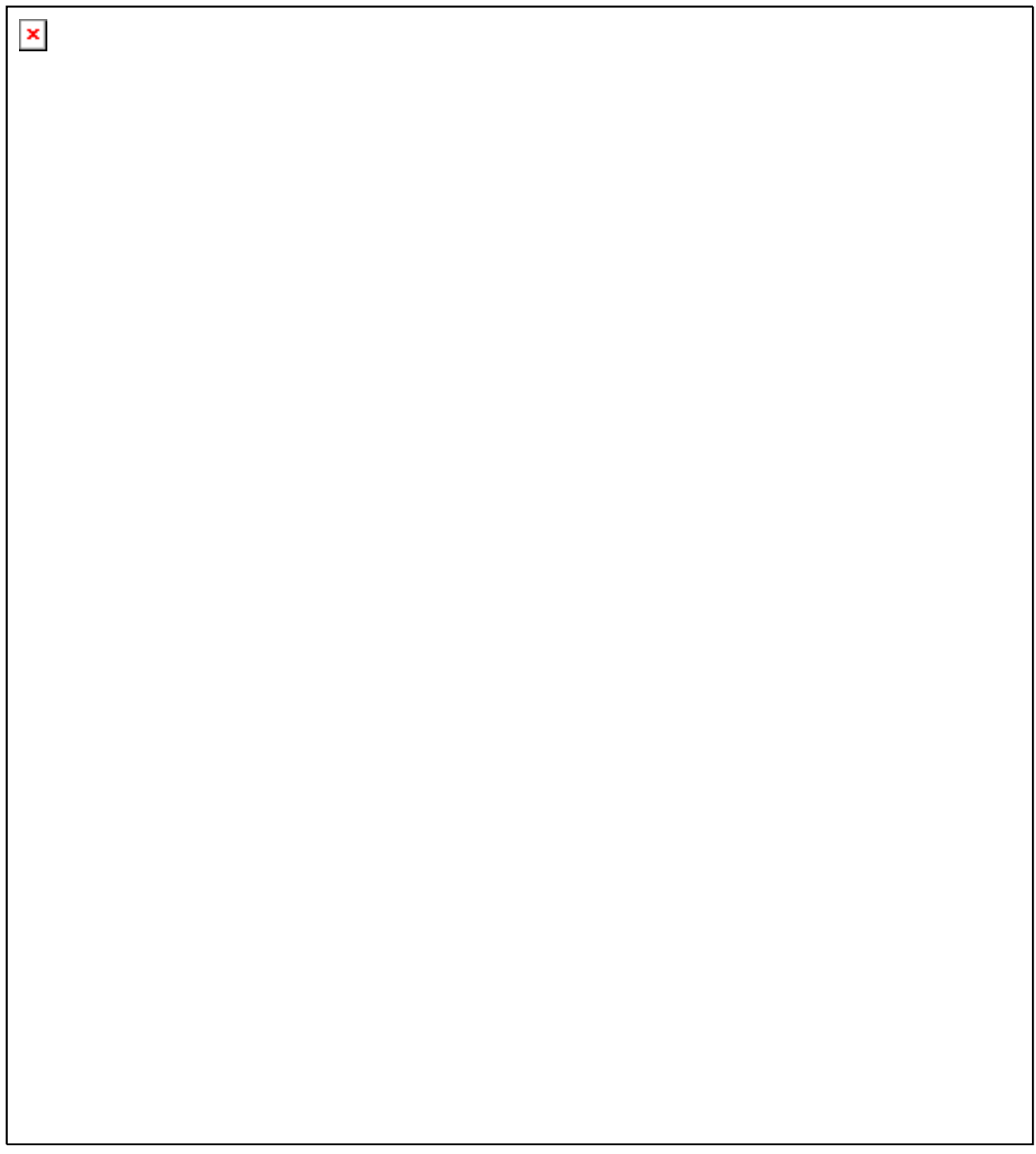

Figure 7 $200 \times 221 \mathrm{~mm}(600 \times 600 \mathrm{DPI})$ 


1
2
3
4
5
6
7
8
9
10
11
12
13
14
15
16
17
18
19
20
21
22
23
24
25
26
27
28
29
30
31
32
33
34
35
36
37
38
39
40
41
42
43
40
45
49
50
51
52
53
55
50

$$
x
$$

Figure 8 $201 \times 270 \mathrm{~mm}(600 \times 600 \mathrm{DPI})$ 
Figure 9 $291 \times 281 \mathrm{~mm}(500 \times 500$ DPI $)$ 


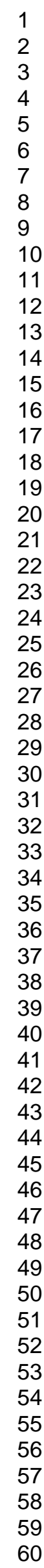

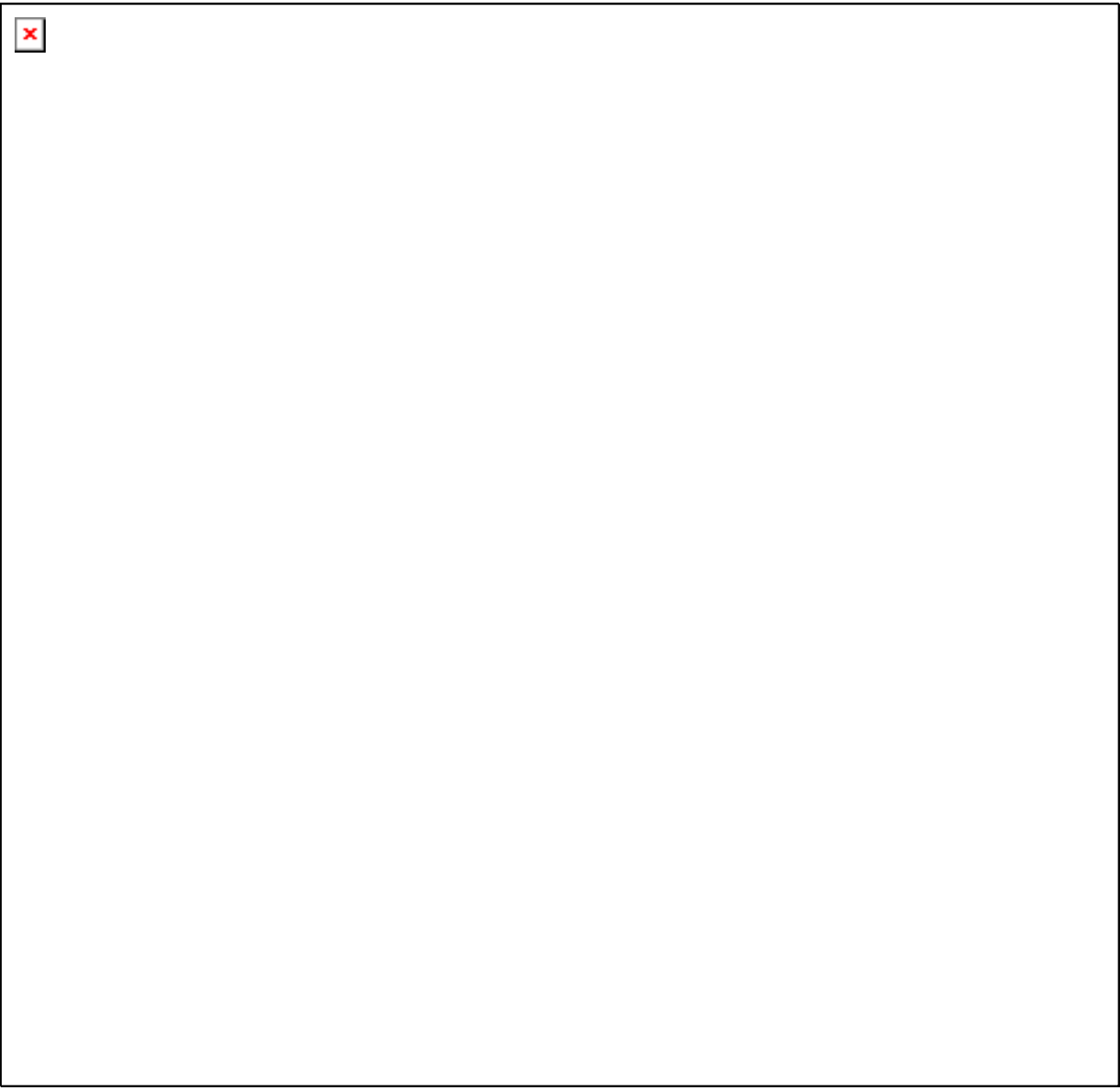

Figure 10

$291 \times 281 \mathrm{~mm}(500 \times 500$ DPI $)$

http://mc.manuscriptcentral.com/pm-pml 


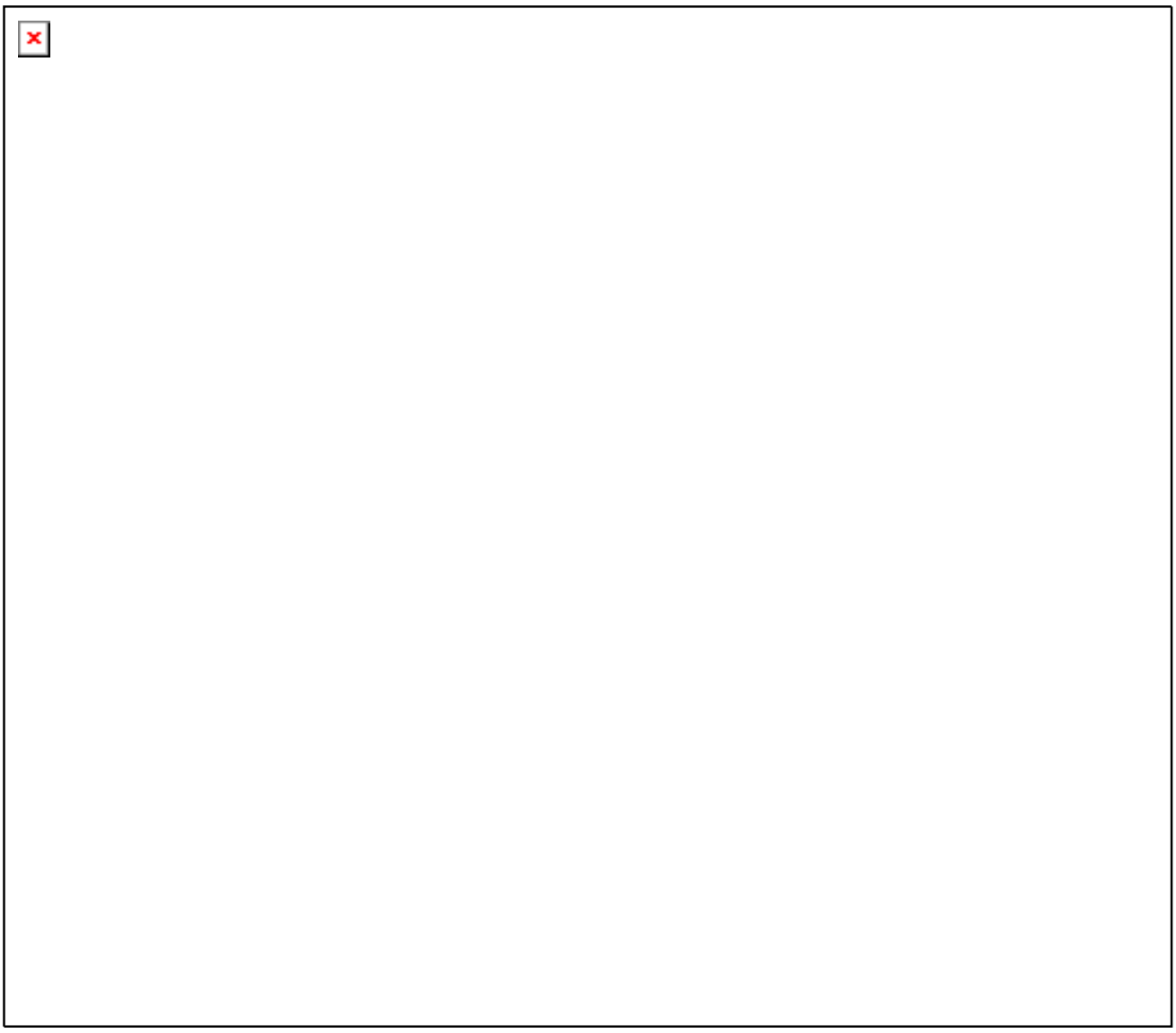

Figure 11

$242 \times 212 \mathrm{~mm}(600 \times 600 \mathrm{DPI})$ 


1
2
3
4
5
6
7
8
9
10
11
12
13
14
15
16
17
18
19
20
21
22
23
24
25
26
27
28
29
30
31
32
33
34
35
36
37
38
39
40
41
42
43
44
45
46
47
48
49
50
51
52
53
54
55
56
57
58
60

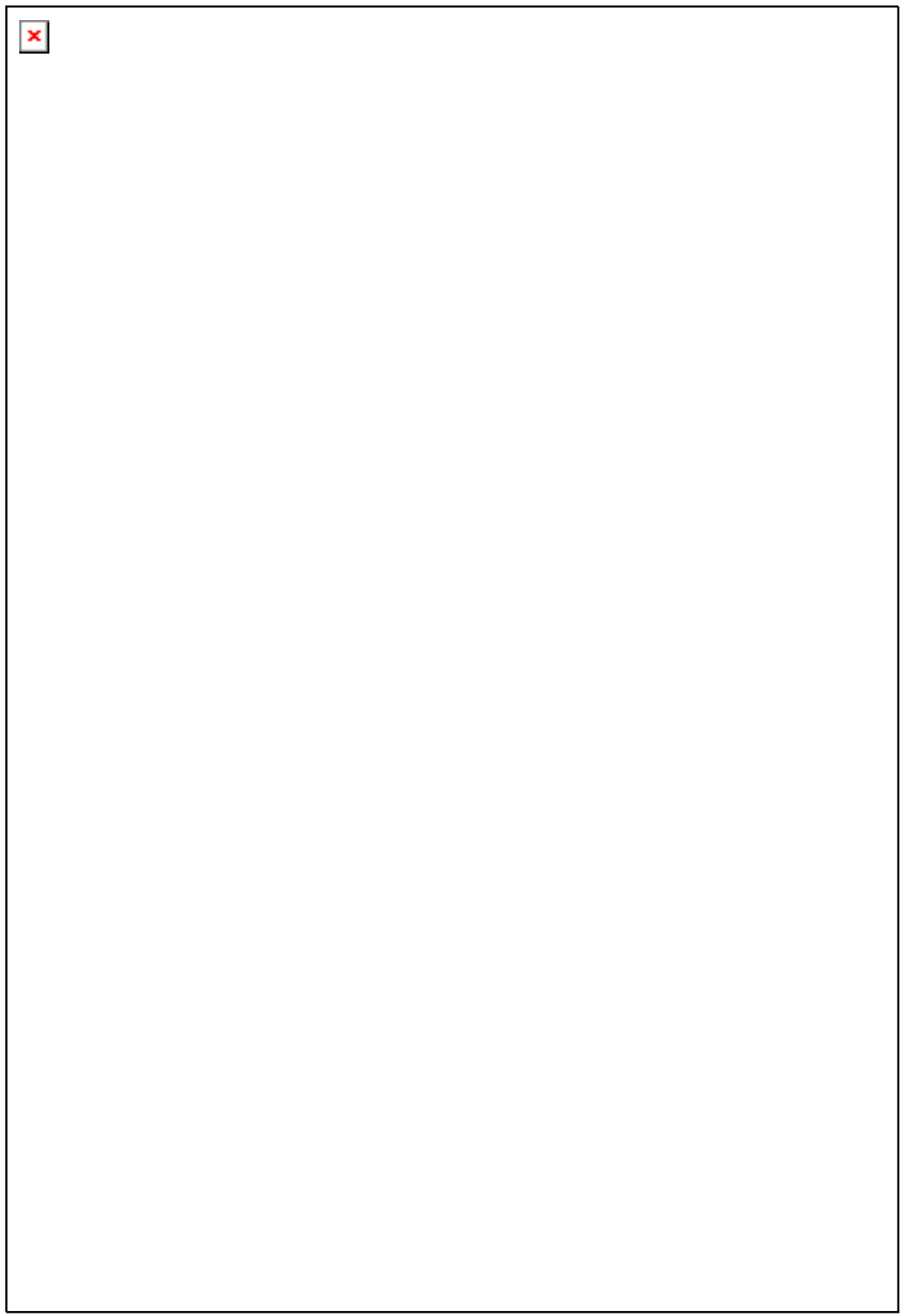

Figure 12

$161 \times 235 \mathrm{~mm}(600 \times 600 \mathrm{DPI})$ 


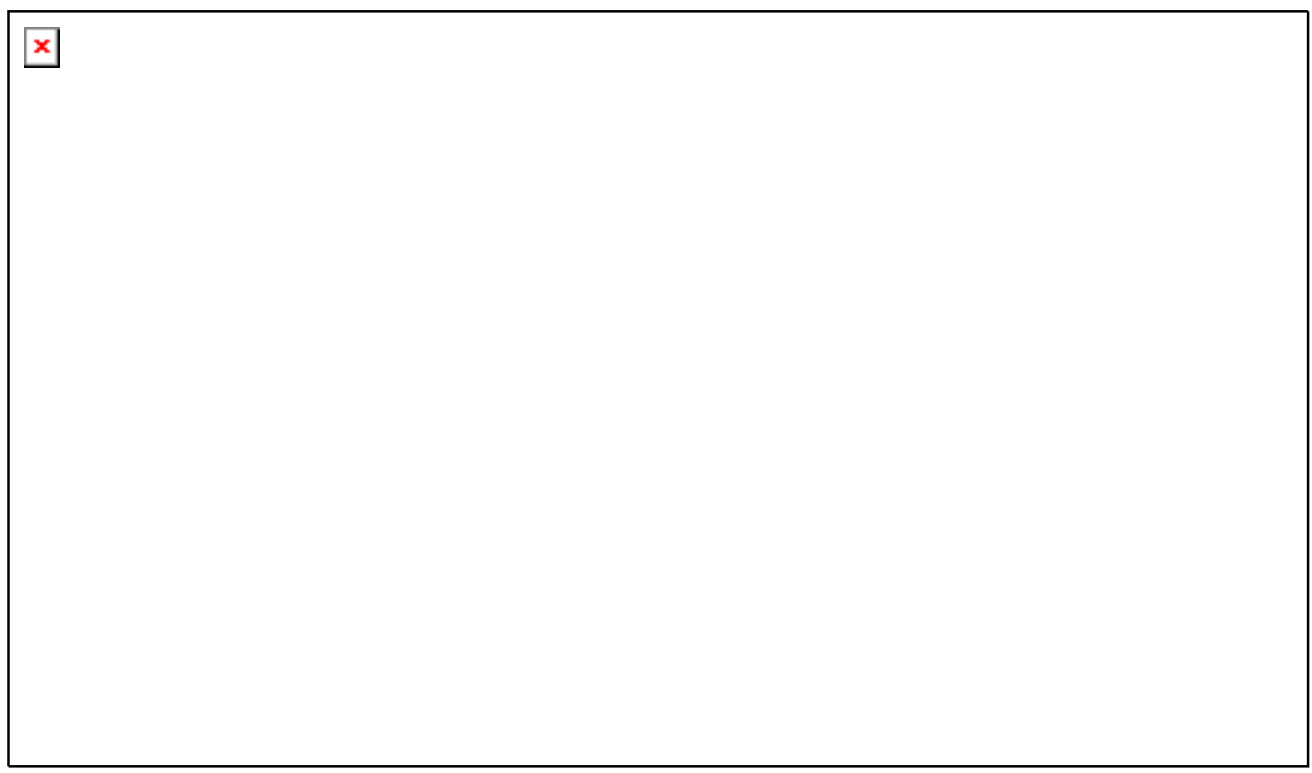

Figure 13

$359 \times 209 \mathrm{~mm}(300 \times 300 \mathrm{DPI})$ 